\title{
Numerical Solution of Fuzzy Delay Differential Equations by Fifth Order Runge-Kutta Method
}

\author{
T.Muthukumar ${ }^{1}$, T.Jayakumar ${ }^{2}$, D.Prasantha Bharathi ${ }^{2 *}$ \\ ${ }^{1}$ Department of Science and Humanities \\ Assistant Professor \\ Dhaanish Ahmed institute of Technology \\ Coimbatore-641 105, Tamilnadu, India \\ ${ }^{2}$ Department of Mathematics \\ Assistant Professor \\ $2 *$ Research Scholar \\ Sri Ramakrishna Mission Vidyalaya College of Arts and Science \\ Coimbatore-641 020, Tamilnadu, India \\ vtmuthukumar@gmail.com, jayakumar.thippan68@gmail.com
}

\begin{abstract}
:
In this paper, we develop the numerical solutions of certain type called Fuzzy Delay Differential Equations(FDE) by using fifth order Runge-Kutta method for fuzzy differential equations. This method based on the seikkala derivative and finally we discuss the numerical examples to illustrate the theory.
\end{abstract}

Keywords : Fuzzy Differential Equations; Fuzzy Delay Differential Equations; Runge-Kutta Method of order five.

\section{AMS Classification:65XXX}

\section{Introduction}

The Delay differential equations are considered as a branch of ordinary differential equations its arise to describe the same physical phenomena, but they are different. The delay diffferential equations is the derivatives of unknown functions are dependent on the values of the functions at previous time. The concept of fuzzy derivative was first introduced by Chang, Zadeh in [7] it was followed up by Dubois, Prede in [9], who defined and used the extension principle. The study of fuzzy differential equations has been growing in recent years and has may application in science and engineering. The numerical method for solving fuzzy differential equations is introduced by Ma, Friedmen, Kandedl in [16] by the standard Euler method and by authors in [1, 2] by Taylor method. In the last few years many works have been performed by several authors in numerical solutions of fuzzy differential equations $[1,2,3,4,5,6]$. D.Prasantha Bharathi and T.Jayakumar discussed Numerical Solution of fuzzy pure multiple Neutral Delay Differential Equations using Runge Kutta method [17]. Alfredo Bellan and Marino Zennaro studied numerical methods for delay differential equations in detail. D.Prasantha Bharathi et.al studied Exixtence and uniqueness of solution for Fuzzy Mixed type of Delay differential equations [18]. D.Prasantha Bhrathi and Jayakumar investigate different type of fuzzy Delay differential Equations with examples [14, 15, 17, 18, 19]. Also D.Prasantha Bharathi et.al discussed numerical solution of fuzzy multiple hybrid single neutral delay differential equations [20] .

Abbasbandy and Allahviranloo [3] discussed a numerical method for solving fuzzy differential equation by Runge-Kutta method of order four. Pederson and Sambandham [21] have investigated the numerical solution of hybrid fuzzy differential equation by using Runge-Kutta method. AlRawi et all [23] have discussed a numerical method for solving Delay differential equations by Runge-Kutta method of order four.

In this article, we develop numerical method for addressing fuzzy delay differential equation by an application of the Runge-Kutta method of order four [23]. In Section 2 we discuss about the Fuzzy Delay Differential Equations(FDDE's). In Section 3 the R-K method of order five for 
approaching fuzzy delay differential equations is discussed. Section 4 contains numerical examples to illustrate the theory.

\section{Fuzzy Delay Differential Equations (FDDE)}

Let us consider the FDDE

$$
\left\{\begin{array}{l}
y^{\prime}(t)=f(t, y(t), y(t-\tau)), \quad t \geq 0 \\
y(t)=\phi(t), \quad-\tau \leq t \leq 0 \\
y\left(t_{0}\right)=y_{0} \in \phi(t)
\end{array}\right.
$$

where $f:[0, \infty) \times R \times R \rightarrow E^{n}$ and $\phi \in R$ is a continuous fuzzy mapping and the initial condition $y_{0} \in \phi$ then $y_{0}(s)=y(s)=\phi(s), \quad-\tau \leq s \leq 0$. Also $y_{0}$ is a fuzzy number with $\alpha$-level intervals $\left[y_{0}\right]^{\alpha}=\left[\underline{y}_{0}^{\alpha}, \bar{y}_{0}^{\alpha}\right], \quad 0 \leq \alpha \leq 1$. The extension principle of Zadeh leads to the following definition of $g(t, y(\bar{t}), y(t-\tau))$ when $y$ is a fuzzy number.

It follows that

$g(t, y(t), y(t-\tau))=\left\{\begin{array}{l}\min g(t, u(t), v(t-\tau)): u(t) \in\left(\underline{y}(t)^{\alpha}, \bar{y}(t)^{\alpha}\right), v(t-\tau) \in\left(\underline{y}(t-\tau)^{\alpha}, \bar{y}(t-\tau)^{\alpha}\right), \\ \max g\left(t, u(t), v(t-\tau): u(t) \in\left(\underline{y}(t)^{\alpha}, \bar{y}(t)^{\alpha}\right), v(t-\tau) \in\left(\underline{y}(t-\tau)^{\alpha}, \bar{y}(t-\tau)^{\alpha}\right)\right.\end{array}\right.$

for $y \in E$ with $\alpha$ - level sets $[y]_{\alpha}=\left[\underline{y}^{\alpha}, \bar{y}^{\alpha}\right], 0<\alpha \leq 1$

Since the fuzzy derivative $y^{\prime}(t)$ of a fuzzy process, $y: R_{+} \rightarrow E$ is defined by $\left[y^{\prime}(t)\right]_{\alpha}=\left[\left(\underline{y}^{\alpha}\right)^{\prime}(t),\left(\bar{y}^{\alpha}\right)^{\prime}(t)\right]$, $0<\alpha \leq 1$.

We call $y: R_{+} \rightarrow E$ a fuzzy solution of (5) on the interval $I=[0, T]$ if

$$
\left\{\begin{array}{l}
\left(\underline{y}^{\alpha}\right)^{\prime}(t)=\min f(t, u(t), v(t-\tau)): u(t) \in\left(\underline{y}(t)^{\alpha}, \bar{y}(t)^{\alpha}\right), v(t-\tau) \in\left(\underline{y}(t-\tau)^{\alpha}, \bar{y}(t-\tau)^{\alpha}\right), \\
\left(\bar{y}^{\alpha}\right)^{\prime}(t)=\max f\left(t, u(t), v(t-\tau): u(t) \in\left(\underline{y}(t)^{\alpha}, \bar{y}(t)^{\alpha}\right), v(t-\tau) \in\left(\underline{y}(t-\tau)^{\alpha}, \bar{y}(t-\tau)^{\alpha}\right),\right.
\end{array}\right.
$$

for $t \in I$ and $0<\alpha \leq 1$.

\section{Definition 2.1 [8]}

Let $I$ be a real interval and $F: I \rightarrow E^{n}$. If, for arbitrary fixed $t_{0} \in I$ and $\epsilon>0$, there exist $\delta>0$, (depending on $t_{0}$ and $\epsilon$ ) such that

$$
t \in I, \quad\left|t-t_{0}\right|<\delta \Rightarrow D\left(F(t), F\left(t_{0}\right)\right)<\epsilon,
$$

then $F$ is said to be continuous on $I$.

If $J=[a, b]$ is compact a compact interval in $E$, then $C\left(J, E^{n}\right)$ represents the set of all continuous fuzzy functions from $J$ into $E^{n}$. In the space $C\left(J, E^{n}\right)$, we consider the following metric:

$$
D(u, v)=\sup _{t \in J} D[u(t), v(t)]
$$

Following the notation in [?], for a positive number $\tau$, we denote by $C_{\tau}$, the space $C\left([-\tau, 0], E^{n}\right)$, equipped with the metric defined by

$$
D_{\tau}(u, v)=\sup _{t \in[-\tau, 0]} D[u(t), v(t)] .
$$

Remaining faithful to the classical notation used in the field of functional differential equations [10], for a given $u \in C\left([-\tau, \infty], E^{n}\right), u_{t}$ denotes for each $t \in[0, \infty)$, the element in $C_{\tau}$, defined by

$$
u_{t}(s)=u(t+s), s \in[-\tau, 0] .
$$

\section{Lemma 2.1}

If $g:[0, \infty) \times R \times R \rightarrow E^{n}$ is a jointly continuous function and $u:[-\tau, \infty) \rightarrow E^{n}$ is a continuous function, then the function

$$
t \in[0, \infty) \rightarrow F(t, u(t), v(t-\tau)) \in E^{n}
$$


is also continuous.

\section{Theorem 2.1}

Let $g:[0, \infty) \times R \times R \rightarrow E^{n}$ be a continuous fuzzy function such that there exists $\mathrm{K}$ and $M>0$ such that $\left|\left(f\left(t, u_{1}, v_{1}\right)-f\left(t, u_{2}, v_{2}\right)\right)\right| \leq K\left|u_{1}-u_{2}\right|+M\left|v_{1}-v_{2}\right|$, for all $t \in[0, \infty), u_{1}, u_{2}, v_{1}, v_{2} \in E^{n}$. Then (5) has a solution on I.

\section{Fifth order Runge - Kutta Method}

Here we consider for a FDDE's from equation (1), to constract the fuzzy delay differential equations (FDDE) via an application of Runge-Kutta method for fuzzy differential equation [3] using the method of Runge-Kutta method of order five when $g$ in (1) can be obtained via the Zadeh extension principle from $g \in C\left[R^{+} \times R \times R, R\right]$. We assume that the existence and uniqueness of solutions of (1) hold for each $\left[t_{k}, t_{k+1}\right]$.

This Runge - Kutta method is the fifth order approximation of $\underline{Y}_{k}^{\prime}(t ; \alpha)$ and $\bar{Y}_{k}^{\prime}(t ; \alpha)$. We define

$$
\begin{aligned}
& \underline{X}\left(t_{n+1} ; \alpha\right)-\underline{X}\left(t_{n} ; \alpha\right)=\sum_{i=1}^{6} w_{i} \underline{K}_{i}\left(t_{n} ; x\left(t_{n} ; \alpha\right)\right), \\
& \bar{X}\left(t_{n+1} ; \alpha\right)-\bar{X}\left(t_{n} ; \alpha\right)=\sum_{i=1}^{6} w_{i} \bar{K}_{i}\left(t_{n} ; x\left(t_{n} ; \alpha\right)\right),
\end{aligned}
$$

where $w_{1}, w_{2}, w_{3}, w_{5}, w_{5}$ and $w_{6}$ are constants and

$$
\begin{aligned}
& \underline{K}_{1}(t ; x(t ; \alpha))=\min \{h g(t, u(t), v(t-\tau)) \mid u(t) \in[\underline{x}(t ; \alpha), \bar{x}(t ; \alpha)], v(t-\tau) \in[\underline{x}(t-\tau ; \alpha), \bar{x}(t-\tau ; \alpha)]\}, \\
& \bar{K}_{1}(t ; x(t ; \alpha))=\max \{h g(t, u(t), v(t-\tau)) \mid u(t) \in[\underline{x}(t ; \alpha), \bar{x}(t ; \alpha)], v(t-\tau) \in[\underline{x}(t-\tau ; \alpha), \bar{x}(t-\tau ; \alpha)]\}, \\
& \underline{K}_{2}(t ; x(t ; \alpha))=\min \left\{h g\left(t+\frac{h}{2}, u(t), v(t-\tau)\right) \mid u(t) \in\left[\underline{z}_{1}(t, x(t ; \alpha)), \bar{z}_{1}(t, x(t ; \alpha))\right],\right. \\
& \left.v(t-\tau) \in\left[\underline{z}_{1}(t-\tau, x(t-\tau ; \alpha)), \bar{z}_{1}(t-\tau, x(t-\tau ; \alpha))\right]\right\}, \\
& \bar{K}_{2}(t ; x(t ; \alpha))=\max \left\{h g\left(t+\frac{h}{2}, u(t), v(t-\tau)\right) \mid u(t) \in\left[\underline{z}_{1}(t, x(t ; \alpha)), \bar{z}_{1}(t, x(t ; \alpha))\right],\right. \\
& \left.v(t-\tau) \in\left[\underline{z}_{1}(t-\tau, x(t-\tau ; \alpha)), \bar{z}_{1}(t-\tau, x(t-\tau ; \alpha))\right]\right\} \\
& \underline{K}_{3}(t ; x(t ; \alpha))=\min \left\{h g\left(t+\frac{h}{4}, u(t), v(t-\tau)\right) \mid u(t) \in\left[\underline{z}_{2}(t, x(t ; \alpha)), \bar{z}_{2}(t, x(t ; \alpha))\right],\right. \\
& \left.v(t-\tau) \in\left[\underline{z}_{2}(t-\tau, x(t-\tau ; \alpha)), \bar{z}_{2}(t-\tau, x(t-\tau ; \alpha))\right]\right\} \\
& \bar{K}_{3}(t ; x(t ; \alpha))=\max \left\{h g\left(t+\frac{h}{4}, u(t), v(t-\tau)\right) \mid u(t) \in\left[\underline{z}_{2}(t, x(t ; \alpha)), \bar{z}_{2}(t, x(t ; \alpha))\right],\right. \\
& \left.v(t-\tau) \in\left[\underline{z}_{2}(t-\tau, x(t-\tau ; \alpha)), \bar{z}_{2}(t-\tau, x(t-\tau ; \alpha))\right]\right\}, \\
& \underline{K}_{4}(t ; x(t ; \alpha))=\min \left\{h g\left(t+\frac{h}{2}, u(t), v(t-\tau)\right) \mid u(t) \in\left[\underline{z}_{3}(t, x(t ; \alpha)), \bar{z}_{3}(t, x(t ; \alpha))\right],\right. \\
& \left.v(t-\tau) \in\left[\underline{z}_{3}(t-\tau, x(t-\tau ; \alpha)), \bar{z}_{3}(t-\tau, x(t-\tau ; \alpha))\right]\right\},
\end{aligned}
$$




$$
\begin{aligned}
\bar{K}_{4}(t ; x(t ; \alpha))= & \max \left\{h g\left(t+\frac{h}{2}, u(t), v(t-\tau)\right) \mid u(t) \in\left[\underline{z}_{3}(t, x(t ; \alpha)), \bar{z}_{3}(t, x(t ; \alpha))\right],\right. \\
& \left.v(t-\tau) \in\left[\underline{z}_{3}(t-\tau, x(t-\tau ; \alpha)), \bar{z}_{3}(t-\tau, x(t-\tau ; \alpha))\right]\right\}, \\
\underline{K}_{5}(t ; x(t ; \alpha))= & \min \left\{h g\left(t+\frac{3 h}{2}, u(t), v(t-\tau)\right) \mid u(t) \in\left[\underline{z}_{4}(t, x(t ; \alpha)), \bar{z}_{4}(t, x(t ; \alpha))\right],\right. \\
& \left.v(t-\tau) \in\left[\underline{z}_{4}(t-\tau, x(t-\tau ; \alpha)), \bar{z}_{4}(t-\tau, x(t-\tau ; \alpha))\right]\right\}, \\
\bar{K}_{5}(t ; x(t ; \alpha))= & \max \left\{h g\left(t+\frac{3 h}{2}, u(t), v(t-\tau)\right) \mid u(t) \in\left[\underline{z}_{4}(t, x(t ; \alpha)), \bar{z}_{4}(t, x(t ; \alpha))\right],\right. \\
& \left.v(t-\tau) \in\left[\underline{z}_{4}(t-\tau, x(t-\tau ; \alpha)), \bar{z}_{4}(t-\tau, x(t-\tau ; \alpha))\right]\right\}, \\
\underline{K}_{6}(t ; x(t ; \alpha))= & \min \left\{h g(t+h, u(t), v(t-\tau)) \mid u(t) \in\left[\underline{z}_{5}(t, x(t ; \alpha)), \bar{z}_{5}(t, x(t ; \alpha))\right],\right. \\
& \left.v(t-\tau) \in\left[\underline{z}_{5}(t-\tau, x(t-\tau ; \alpha)), \bar{z}_{5}(t-\tau, x(t-\tau ; \alpha))\right]\right\}, \\
& \left.v(t-\tau) \in\left[\underline{z}_{5}(t-\tau, x(t-\tau ; \alpha)), \bar{z}_{5}(t-\tau, x(t-\tau ; \alpha))\right]\right\},
\end{aligned}
$$

Next we define

$$
\begin{aligned}
& \underline{z}_{1}(t, x(t ; \alpha))=\underline{x}(t ; \alpha)+\frac{1}{2} \underline{K}_{1}(t, x(t ; \alpha)), \\
& \bar{z}_{1}(t, x(t ; \alpha))=\bar{x}(t ; \alpha)+\frac{1}{2} \bar{K}_{1}(t, x(t ; \alpha)), \\
& \underline{z}_{2}(t, x(t ; \alpha))=\underline{x}(t ; \alpha)+\frac{3}{16} \underline{K}_{2}(t, x(t ; \alpha))+\frac{1}{16} \underline{K}_{2}(t, x(t ; \alpha)), \\
& \bar{z}_{2}(t, x(t ; \alpha))=\bar{x}(t ; \alpha)+\frac{3}{16} \bar{K}_{1}(t, x(t ; \alpha))+\frac{1}{16} \bar{K}_{2}(t, x(t ; \alpha)), \\
& \underline{z}_{3}(t, x(t ; \alpha))=\underline{x}(t ; \alpha)+\frac{1}{2} \underline{K}_{3}(t, x(t ; \alpha)), \\
& \bar{z}_{3}(t, x(t ; \alpha))=\bar{x}(t ; \alpha)+\frac{1}{2} \bar{K}_{3}(t, x(t ; \alpha)), \\
& \underline{z}_{4}(t, x(t ; \alpha))=\underline{x}(t ; \alpha)-\frac{3}{16} \underline{K}_{2}(t, x(t ; \alpha))+\frac{6}{16} \underline{K}_{3}(t, x(t ; \alpha))+\frac{9}{16} \underline{K}_{3}(t, x(t ; \alpha)), \\
& \bar{z}_{4}(t, x(t ; \alpha))=\bar{x}(t ; \alpha)-\frac{3}{16} \bar{K}_{2}(t, x(t ; \alpha))+\frac{6}{16} \bar{K}_{3}(t, x(t ; \alpha))+\frac{9}{16} \bar{K}_{4}(t, x(t ; \alpha)), \\
& \underline{z}_{5}(t, x(t ; \alpha))=\underline{x}(t ; \alpha)+\frac{1}{7} \underline{K}_{1}(t, x(t ; \alpha))+\frac{4}{7} \underline{K}_{2}(t, x(t ; \alpha))+\frac{6}{7} \underline{K}_{3}(t, x(t ; \alpha))-\frac{12}{7} \underline{K}_{4}(t, x(t ; \alpha))+\frac{8}{7} \underline{K}_{5}(t, x(t ; \alpha)), \\
& \bar{z}_{5}(t, x(t ; \alpha))=\bar{x}(t ; \alpha)+\frac{1}{7} \bar{K}_{1}(t, x(t ; \alpha))+\frac{4}{7} \bar{K}_{2}(t, x(t ; \alpha))+\frac{6}{7} \bar{K}_{3}(t, x(t ; \alpha))-\frac{12}{7} \bar{K}_{4}(t, x(t ; \alpha))+\frac{8}{7} \bar{K}_{5}(t, x(t ; \alpha)) .
\end{aligned}
$$

Next we define

$S[(t, \underline{x}(t ; \alpha), \bar{x}(t ; \alpha))]=7 \underline{K}_{1}(t, x(t ; \alpha))+32 \underline{K}_{3}(t, x(t ; \alpha))+12 \underline{K}_{4}(t, x(t ; \alpha))+32 \underline{K}_{5}(t, x(t ; \alpha))+7 \underline{K}_{6}(t, x(t ; \alpha))$, $T[(t, \underline{x}(t ; \alpha), \bar{x}(t ; \alpha))]=7 \bar{K}_{1}(t, x(t ; \alpha))+32 \bar{K}_{3}(t, x(t ; \alpha))+12 \bar{K}_{4}(t, x(t ; \alpha))+32 \bar{K}_{5}(t, x(t ; \alpha))+7 \bar{K}_{6}(t, x(t ; \alpha))$.

The exact solution at $t_{n+1}$ is given by

$$
\left\{\begin{array}{l}
\underline{X}\left(t_{n+1} ; \alpha\right) \approx \underline{X}\left(t_{n} ; \alpha\right)+\frac{1}{90} S\left[\left(t_{n}, \underline{X}\left(t_{n} ; \alpha\right), \bar{X}_{n}(t ; \alpha)\right)\right] \\
\bar{X}\left(t_{n+1} ; \alpha\right) \approx \bar{X}\left(t_{n} ; \alpha\right)+\frac{1}{90} T\left[\left(t_{n}, \underline{X}\left(t_{n} ; \alpha\right), \bar{X}_{n}(t ; \alpha)\right)\right] .
\end{array}\right.
$$


The approximate solution is given by

$$
\left\{\begin{array}{l}
\underline{x}\left(t_{n+1} ; \alpha\right)=\underline{x}\left(t_{n} ; \alpha\right)+\frac{1}{90} S\left[\left(t_{n}, \underline{x}\left(t_{n} ; \alpha\right), \bar{x}_{n}(t ; \alpha)\right)\right] \\
\bar{x}\left(t_{n+1} ; \alpha\right)=\bar{x}\left(t_{n} ; \alpha\right)+\frac{1}{90} T\left[\left(t_{n}, \underline{x}\left(t_{n} ; \alpha\right), \bar{x}_{n}(t ; \alpha)\right)\right] .
\end{array}\right.
$$

\section{Theorem 3.1}

Consider the systems (5) and (9). For a fixed $\alpha \in[0,1]$,

$$
\begin{aligned}
& \lim _{h \rightarrow 0} \underline{X}\left(t_{N} ; \alpha\right)=\underline{X}\left(t_{N} ; \alpha\right), \\
& \lim _{h \rightarrow 0} \bar{X}\left(t_{N} ; \alpha\right)=\bar{X}\left(t_{N} ; \alpha\right),
\end{aligned}
$$

\section{Numerical Examples}

Consider the FDDE

$$
\left\{\begin{array}{l}
x^{\prime}(t)=g(t, x(t), x(t-\tau)), \quad t \geq 0 \\
x(t)=\phi(t), \quad-\tau \leq t \leq 0
\end{array}\right.
$$

where $\phi(t)$ be a initial function

$$
[x(t)]^{\alpha}=[\underline{x}(t ; \alpha), \bar{x}(t ; \alpha)], \quad t \geq 0, \quad[\phi(t)]^{\alpha}=[\underline{\phi}(t ; \alpha), \bar{\phi}(t ; \alpha)], \quad t \in[-\tau, 0]
$$

and

$$
\begin{aligned}
{[g(t, x(t), x(t-\tau))]^{\alpha}=\quad } & {[\underline{g}(t, \underline{x}(t ; \alpha), \bar{x}(t ; \alpha), \underline{x}(t-\tau ; \alpha), \bar{x}(t-\tau ; \alpha)),} \\
& \bar{g}(t, \underline{x}(t ; \alpha), \bar{x}(t ; \alpha), \underline{x}(t-\tau ; \alpha), \bar{x}(t-\tau ; \alpha))], \quad t \geq 0
\end{aligned}
$$

\section{Example 4.1}

Consider the FDDE

$$
\left\{\begin{array}{l}
x^{\prime}(t)=x(t)+x(t-1), \quad t \geq 0, \\
x(t)=\phi(t), \quad-1 \leq t \leq 0
\end{array}\right.
$$

Let $\phi(t)=[(0.75+0.25 \alpha),(1.125-0.125 \alpha)] . \alpha \in[0,1]$

By using fifth order Runge-Kutta method we have,

for $t \in[0,1]$

$$
\begin{aligned}
\underline{x}\left(\frac{i}{10} ; \alpha\right)= & \underline{x}\left(\frac{i-1}{10} ; \alpha\right)\left(1+h+\frac{h^{2}}{2}+\frac{h^{3}}{6}+\frac{h^{4}}{24}+\frac{h^{5}}{120}+\frac{h^{6}}{1280}\right) \\
& +\underline{x}\left(\frac{i-1}{10}-1 ; \alpha\right)\left(h+\frac{h^{2}}{2}+\frac{h^{3}}{6}+\frac{h^{4}}{24}+\frac{h^{5}}{120}+\frac{h^{6}}{1280}\right), \\
\bar{x}\left(\frac{i}{10} ; \alpha\right)= & \bar{x}\left(\frac{i-1}{10} ; \alpha\right)\left(1+h+\frac{h^{2}}{2}+\frac{h^{3}}{6}+\frac{h^{4}}{24}+\frac{h^{5}}{120}+\frac{h^{6}}{1280}\right) \\
& +\bar{x}\left(\frac{i-1}{10}-1 ; \alpha\right)\left(h+\frac{h^{2}}{2}+\frac{h^{3}}{6}+\frac{h^{4}}{24}+\frac{h^{5}}{120}+\frac{h^{6}}{1280}\right),
\end{aligned}
$$

where $i=1,2, \cdots 10$.

For $t \in[1,2]$

$$
\underline{x}\left(1+\frac{i}{10} ; \alpha\right)=\underline{x}\left(1+\frac{i-1}{10} ; \alpha\right)\left(1+h+\frac{h^{2}}{2}+\frac{h^{3}}{6}+\frac{h^{4}}{24}+\frac{h^{5}}{120}+\frac{h^{6}}{1280}\right)
$$




$$
\begin{aligned}
& +\underline{x}\left(\frac{i-1}{10} ; \alpha\right)\left(h+\frac{h^{2}}{2}+\frac{h^{3}}{6}+\frac{h^{4}}{24}+\frac{h^{5}}{120}+\frac{h^{6}}{1280}\right) \\
& +\underline{x}\left(\frac{i-1}{10} ; \alpha\right)\left(\frac{h^{2}}{2}+\frac{h^{3}}{3}+\frac{h^{4}}{8}+\frac{h^{5}}{30}+\frac{h^{6}}{256}\right)+\underline{x}\left(\frac{i-1}{10}-1 ; \alpha\right)\left(\frac{h^{2}}{2}+\frac{h^{3}}{3}+\frac{h^{4}}{8}+\frac{h^{5}}{30}+\frac{h^{6}}{256}\right), \\
\bar{x}\left(1+\frac{i}{10} ; \alpha\right)= & \bar{y}\left(1+\frac{i-1}{10} ; \alpha\right)\left(1+h+\frac{h^{2}}{2}+\frac{h^{3}}{6}+\frac{h^{4}}{24}+\frac{h^{5}}{120}+\frac{h^{6}}{1280}\right) \\
& +\bar{x}\left(\frac{i-1}{10} ; \alpha\right)\left(h+\frac{h^{2}}{2}+\frac{h^{3}}{6}+\frac{h^{4}}{24}+\frac{h^{5}}{120}+\frac{h^{6}}{1280}\right) \\
+ & \bar{x}\left(\frac{i-1}{10} ; \alpha\right)\left(\frac{h^{2}}{2}+\frac{h^{3}}{3}+\frac{h^{4}}{8}+\frac{h^{5}}{30}+\frac{h^{6}}{256}\right)+\bar{x}\left(\frac{i-1}{10}-1 ; \alpha\right)\left(\frac{h^{2}}{2}+\frac{h^{3}}{3}+\frac{h^{4}}{8}+\frac{h^{5}}{30}+\frac{h^{6}}{256}\right),
\end{aligned}
$$

where $i=1,2, \cdots 10$.

The exact solution of (11) is given by

$X(t ; \alpha)=\left[(0.75+0.25 \alpha)\left(2 e^{t}-1\right),(1.125-0.125 \alpha)\left(2 e^{t}-1\right)\right]$, for $t \in[0,1]$,

$X(t ; \alpha)=\left[(0.75+0.25 \alpha)\left(2\left(e^{t}-2 e^{t-1}+t e^{t-1}\right)+1\right),(1.125-0.125 \alpha)\left(2\left(e^{t}-2 e^{t-1}+t e^{t-1}\right)+1\right)\right]$ for $t \in[1,2]$.

The approximate solution for $t \in[0,2], \alpha \in[0,1]$, is shown in figure 2 . The exact and approximate solution by fifth order Runge-Kutta method are compared and plotted at $\mathrm{t}=2$ in figure 3 and the results of example 5.1 at $\mathrm{t}=2$ are shown in table 1 . The exact solution for $\alpha=1, t \in[0,20]$ is shown in figure 4.

Table 1

Comparison of exact solution and approximate solution by fifth order Runge-Kutta method

\begin{tabular}{|c|r|r|r|r|}
\hline \multirow{2}{*}{$\alpha$} & \multicolumn{2}{|c|}{${\text { R-K } ~^{\text {th }} \text { order }}$} & \multicolumn{2}{|c|}{ Exact Solution } \\
\cline { 2 - 5 } & $\underline{x}\left(t_{i} ; \alpha\right)$ & $\bar{x}\left(t_{i} ; \alpha\right)$ & $\underline{X}\left(t_{i} ; \alpha\right)$ & $\bar{X}\left(t_{i} ; \alpha\right)$ \\
\hline 0 & 11.8335517785545 & 17.7503276678317 & 11.8335841483960 & 17.7503762225940 \\
0.1 & 12.2280035045063 & 17.5531018048558 & 12.2280369533425 & 17.5531498201207 \\
0.2 & 12.6224552304581 & 17.3558759418799 & 12.6224897582890 & 17.3559234176474 \\
0.3 & 13.0169069564099 & 17.1586500789040 & 13.0169425632356 & 17.1586970151742 \\
0.4 & 13.4113586823617 & 16.9614242159281 & 13.4113953681821 & 16.9614706127009 \\
0.5 & 13.8058104083136 & 16.7641983529522 & 13.8058481731286 & 16.7642442102276 \\
0.6 & 14.2002621342654 & 16.5669724899763 & 14.2003009780752 & 16.5670178077544 \\
0.7 & 14.5947138602172 & 16.3697466270004 & 14.5947537830217 & 16.3697914052811 \\
0.8 & 14.9891655861690 & 16.1725207640245 & 14.9892065879682 & 16.1725650028078 \\
0.9 & 15.3836173121208 & 15.9752949010485 & 15.3836593929148 & 15.9753386003346 \\
1 & 15.7780690380726 & 15.7780690380726 & 15.7781121978613 & 15.7781121978613 \\
\hline
\end{tabular}


Approximate solution by fifth order Runge-Kutta method

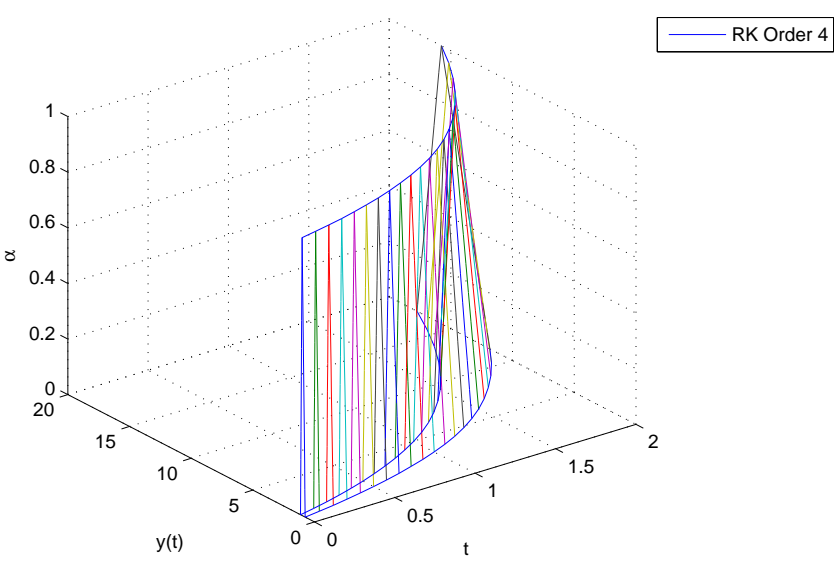

Figure 1: (for $\mathrm{h}=0.1)$

Comparison of exact solution and approximate solution by fifth order R-K method

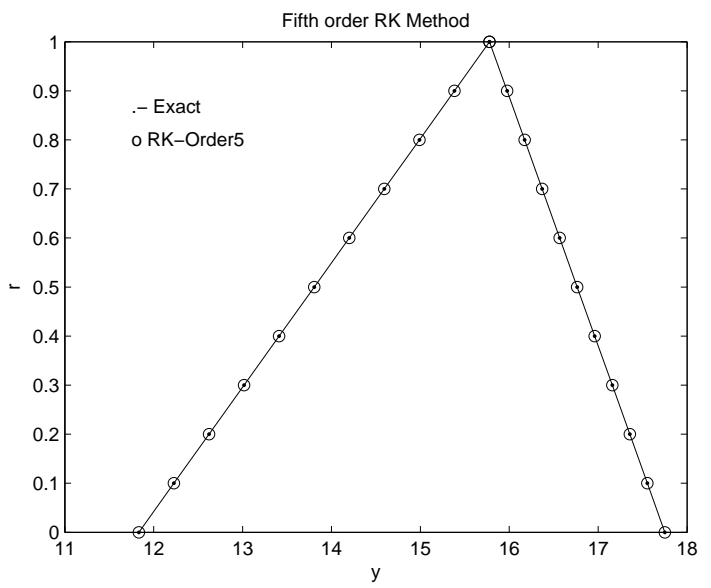

Figure 2: (for $\mathrm{h}=0.1$ and $\mathrm{t}=2$ )

Exact solution for, $\alpha=1, t \in[0,20]$ 


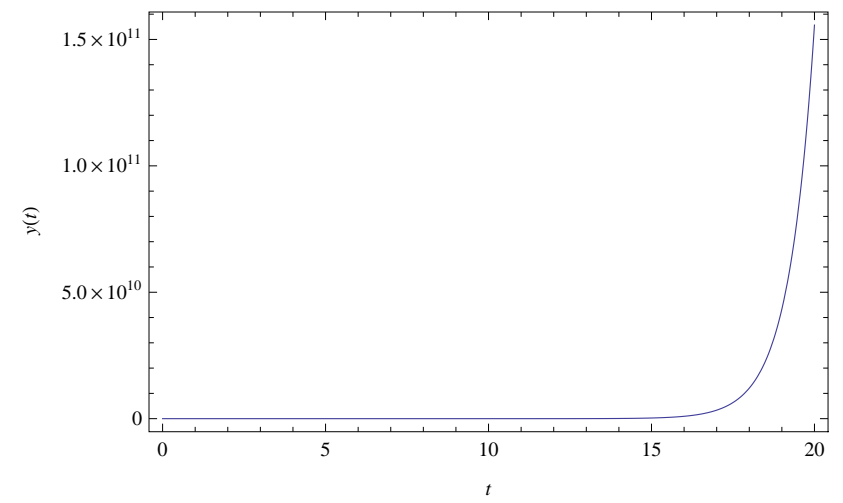

Figure 3:

\section{Example 4.2}

Consider the FDDE

$$
\left\{\begin{array}{l}
x^{\prime}(t)=\lambda x(t-1), \quad t \geq 0 \\
x(t)=\phi(t), \quad-1 \leq t \leq 0
\end{array}\right.
$$

Let $\phi(t)=\left[(0.75+0.25 \alpha) e^{t},(1.125-0.125 \alpha) e^{t}\right]$.

The initial value is given by, $\left[x_{0}\right]^{\alpha}=[0.75+0.25 \alpha, 1.125-0.125 \alpha]$.

The exact solution of (12) is given by,

$$
\begin{aligned}
X(t ; \alpha)= & {\left[(0.75+0.25 \alpha)\left(1+\frac{\lambda\left(e^{t}-1\right)}{e}\right),(1.125-0.125 \alpha)\left(1+\frac{\lambda\left(e^{t}-1\right)}{e}\right)\right], \text { for } t \in[0,1] } \\
X(t ; \alpha)= & {\left[( 0 . 7 5 + 0 . 2 5 \alpha ) \left(1+\lambda\left(t+\lambda \frac{\left(e^{t-1}-t\right)}{e}+\frac{e-1}{e}-1\right),\right.\right.} \\
& (1.125-0.125 \alpha)\left(1+\lambda\left(t+\lambda \frac{\left(e^{t-1}-t\right)}{e}+\frac{e-1}{e}-1\right)\right] . \text { for } t \in[1,2]
\end{aligned}
$$

When $\lambda=-1$ using fifth order Runge-Kutta method we have, for $t \in[0,1]$

$$
\begin{aligned}
& \underline{x}\left(\frac{i}{10} ; \alpha\right)=\underline{x}\left(\frac{i-1}{10} ; \alpha\right)-\underline{x}\left(\frac{i-1}{10}-1 ; \alpha\right)\left(h+\frac{h^{2}}{2}+\frac{h^{3}}{6}+\frac{h^{4}}{24}+\frac{h^{5}}{120}+\frac{h^{6}}{1280}\right), \\
& \bar{x}\left(\frac{i}{10} ; \alpha\right)=\bar{x}\left(\frac{i-1}{10} ; \alpha\right)-\bar{x}\left(\frac{i-1}{10}-1 ; \alpha\right)\left(h+\frac{h^{2}}{2}+\frac{h^{3}}{6}+\frac{h^{4}}{24}+\frac{h^{5}}{120}+\frac{h^{6}}{1280}\right),
\end{aligned}
$$

where $i=1,2, \cdots 10$

for $t \in[1,2]$

$$
\begin{aligned}
\underline{x}\left(1+\frac{i}{10} ; \alpha\right) & =\underline{x}\left(1+\frac{i-1}{10} ; \alpha\right)-h \underline{x}\left(\frac{i-1}{10} ; \alpha\right)+\underline{x}\left(\frac{i-1}{10}-1 ; \alpha\right)\left(\frac{h^{2}}{2}+\frac{h^{3}}{6}+\frac{h^{4}}{24}+\frac{h^{5}}{120}+\frac{h^{6}}{1280}\right), \\
\bar{y}\left(1+\frac{i}{10} ; \alpha\right) & =\bar{y}\left(1+\frac{i-1}{10} ; \alpha\right)-h \bar{x}\left(\frac{i-1}{10} ; \alpha\right)+\bar{x}\left(\frac{i-1}{10}-1 ; \alpha\right)\left(\frac{h^{2}}{2}+\frac{h^{3}}{6}+\frac{h^{4}}{24}+\frac{h^{5}}{120}+\frac{h^{6}}{1280}\right),
\end{aligned}
$$

where $i=1,2, \cdots 10$

When $\lambda=-1$ the exact solution is given by,

$$
\begin{aligned}
& X(t ; \alpha)=\left[(0.75+0.25 \alpha)\left(1-\frac{\left(e^{t}-1\right)}{e}\right),(1.125-0.125 \alpha)\left(1-\frac{\left(e^{t}-1\right)}{e}\right)\right], \text { for } t \in[0,1] \\
& X(t ; \alpha)=[(0.75+0.25 \alpha)\left(1-\left(t-\frac{\left(e^{t-1}-t\right)}{e}+\frac{e-1}{e}-1\right),\right. \\
&(1.125-0.125 \alpha)\left(1-\left(t-\frac{\left(e^{t-1}-t\right)}{e}+\frac{e-1}{e}-1\right)\right], \text { for } t \in[1,2]
\end{aligned}
$$


The approximate solution for $t \in[0,2], \alpha \in[0,1]$, is shown in figure 5 . The exact and approximate solution by fifth order Runge-Kutta method are compared and plotted at $\mathrm{t}=2$ in figure 6 and the results of example 5.2 at $\mathrm{t}=2$ are shown in table 2 . The exact solution for $\alpha=1, t \in[0,20]$ is shown in figure 7.

Table 2

Comparison of exact solution and approximate solution by fifth order Runge-Kutta method

\begin{tabular}{|c|r|r|r|r|}
\hline \multirow{2}{*}{$\alpha$} & \multicolumn{2}{|c|}{ R-K $5^{t h}$ order } & \multicolumn{2}{c|}{ Exact Solution } \\
\cline { 2 - 5 } & $\underline{x}\left(t_{i} ; \alpha\right)$ & $\underline{x}\left(t_{i} ; \alpha\right)$ & $X\left(t_{i} ; \alpha\right)$ & $X\left(t_{i} ; \alpha\right)$ \\
\hline 0 & -0.275909721782563 & -0.413864582673845 & -0.275909580878582 & -0.413864371317873 \\
0.1 & -0.285106712508649 & -0.409266087310802 & -0.285106566907868 & -0.409265878303230 \\
0.2 & -0.294303703234734 & -0.404667591947759 & -0.294303552937154 & -0.404667385288587 \\
0.3 & -0.303500693960820 & -0.400069096584717 & -0.303500538966440 & -0.400068892273944 \\
0.4 & -0.312697684686905 & -0.395470601221674 & -0.312697524995726 & -0.395470399259301 \\
0.5 & -0.321894675412990 & -0.390872105858631 & -0.321894511025012 & -0.390871906244658 \\
0.6 & -0.331091666139076 & -0.386273610495589 & -0.331091497054298 & -0.386273413230014 \\
0.7 & -0.340288656865161 & -0.381675115132546 & -0.340288483083584 & -0.381674920215371 \\
0.8 & -0.349485647591247 & -0.377076619769503 & -0.349485469112870 & -0.377076427200728 \\
0.9 & -0.358682638317332 & -0.372478124406460 & -0.358682455142156 & -0.372477934186085 \\
1 & -0.367879629043418 & -0.367879629043418 & -0.367879441171442 & -0.367879441171442 \\
\hline
\end{tabular}

Approximate solution by fifth order Runge-Kutta method

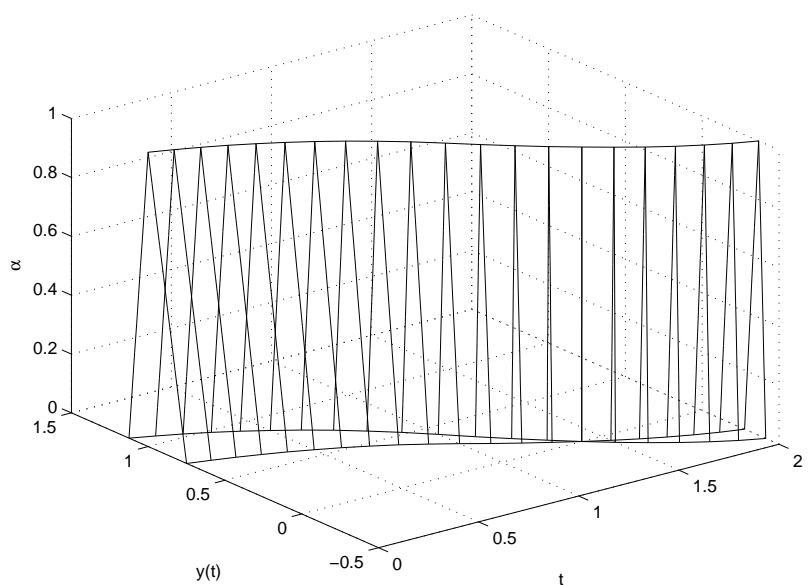

Figure 4: (for $\mathrm{h}=0.1)$ 


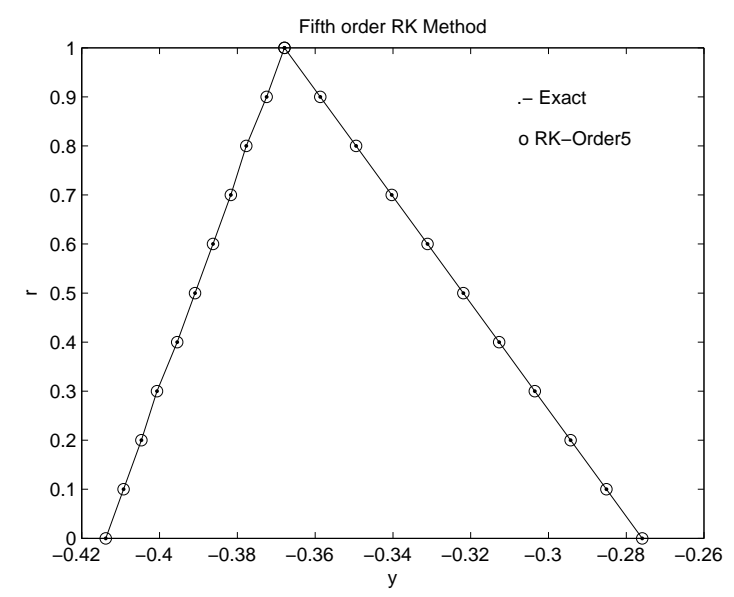

Figure 5: (for $\mathrm{h}=0.1$ and $\mathrm{t}=1$ )

Exact solution for, $\alpha=1, t \in[0,20]$

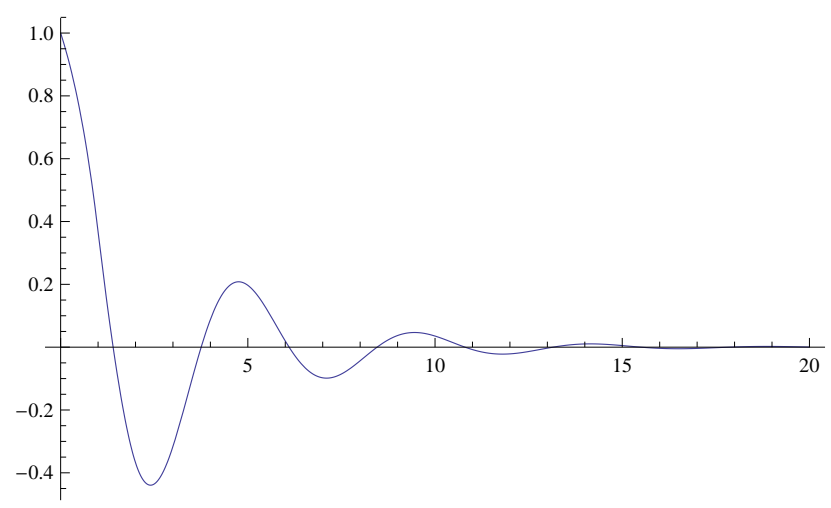

Figure 6:

\section{Conclusion}

In this paper, we presented a numerical iterative solution of fifth order Runge-Kutta method for finding the numerical solution of fuzzy delay differential equations based on Seikkala's derivative and Hukuhara differentiability of fuzzy process are considered. In the proposed method the convergence order is $O\left(h^{5}\right)$.

\section{References}

[1] S. Abbasbandy, T. Allahviranloo, Numerical solution of fuzzy differential equation by Taylor method, Journal of Computational Methods in Applied mathematics, 2 (2002) 113-124.

[2] S. Abbasbandy, T. Allahviranloo, Numerical solution of fuzzy differential equation, Mathematical and Computational Applications, 7 (2002) 41-52.

[3] S. Abbasbandy, T. Allahviranloo, Numerical Solution of fuzzy differential equation by RungeKutta Method, Nonlinear Studies, 11 (2004) 117-129.

[4] T. Allahviranloo, Numerical solution of fuzzy differential equations by Adams-Bashforth twostep method, Journal of Applied Mathematics Islamic Azad University Lahijan, (2004) 36-47. 
[5] T. Allahviranloo, T. Ahmady, E. Ahmady, Numerical solution of fuzzy differential equations by Predictor-Corrector method, Information Sciences, 177 (2007) 1633-1647.

[6] J.J.Bukley, T.Feuring, Fuzzy differential equations, Fuzzy Sets and Systems, 110 (2000), 4354 .

[7] S.L. Chang and L.A. Zadeh, On fuzzy mapping and control, IEEE Transactions on systems Man Cybernetics, 2(1972), 30-34.

[8] P. Diamond, P. Kloeden, 1984. Metric Spaces of Fuzzy sets, World Scientific, Singapore.

[9] D. Dubois, H. Prade, Towards fuzzy differential calculus, Part 3. Differention, Fuzzy Sets and System, 8 (1982), 225-233.

[10] Hale, J. K. 1997. Theory of Functional Differential Equations, Springer, New York.

[11] Jayakumar,T and Kanakarajan,K,(2014) Numerical solution for hybrid fuzzy systems by Runge-Kutta Verner method, Far East Journal of Applied Mathematics, 2, 93-115.

[12] Jayakumar T , Kanagarajan K and Maheshkumar D,(2012), Numerical solution of fuzzy differential equation by Runge-Kutta method of order five, International Journal of Applied Mathematical Science, 6, 2989-3002.

[13] Jayakumar, T and Kanagarajan,K, (2012) Numerical solution for hybrid fuzzy system by Runge-Kutta Fehlberg method, International Journal of Mathematical Analysics, 6, 26192632 .

[14] Muthukumar thangamuthu and Jayakumar T (2019), Numerical Solution for Hybrid Fuzzy Differential equation by Fifth Order Runge-Kutta Nystrom Method,Journal of Mathematical Sciences and Modelling, 2(1) 39-50.

[15] Muthukumar,T, Jayakumar,T and Prasantha Bharathi,D,(2020) Numerical Solution of Fuzzy delay differential Equations by Runge-Kutta Fehlberg Method. GIS Science Journal 7(12).

[16] M. Ma, M. Friedman, A. Kandel, Numerical solutions of fuzzy differential equations, Fuzzy Sets and Systems, 105 (1999) 133-138.

[17] Prasantha Bharathi,D, Jayakumar,T and Vinoth,S(2019) Numerical Solution of Fuzzy Pure Multiple Neutral Delay Differential Equations, International Journal of Advanced Scientific Research Management, 8, 172-178.

[18] Prasantha Bharathi,D, Jayakumar,T, Muthukumar,T and Vinoth,S,(2021) Exixtence and uniqueness of solution for Fuzzy Mixed type of Delay differential equations, Journal of Applied nonlinear Dynamics 10(1), 187-196.

[19] Prasantha Bharathi,D, Jayakumar,T and Vinoth,S, (2019) Numerical Solution of Fuzzy Neutral Delay Differential Equations an application of Runge kutta method of order Four, Journal of Emerging Technology and innovative research6(2).

[20] Prasantha Bharathi,D Jayakumar,T and Vinoth,S, (2019) Numerical Solution of Fuzzy Multiple Hybrid single Neutral Delay Differential Equations, International journal of scientific and Technology research, 8 (9).

[21] S. Pederson and M. Sambandham, The Runge-Kutta method for hybrid fuzzy differential equations, Nonlinear Analysis Hybrid Systems, 2(2008), 626-634.

[22] S. Seikkala, On the fuzzy initial value problem, Fuzzy Sets and Systems, 24 (1987) 319-330.

[23] Suha Najeeb AL Rawi, Raghad Kadhim Salih, Amaal Ali Mohammed, Numerical Solution of $N^{t h}$ order linear delay differential equation using Runge-Kutta method, Um Salama Science journal, Vol 3(1) 2006.140-146. 